\section{Erythema exsudativum multiforme majus mit vorwiegendem Schleimhautbefall (Fuchs-Syndrom) nach Ibuprofen}

Zusammenfassung. Das Fuchs-Syndrom stellt eine Sonderform des Erythema exsudativum multiforme majus (EEMM) mit vorwiegendem Schleimhautbefall dar. Wir berichten über eine 46-jährige Frau, bei der dieses schwere Krankheitsbild durch Ibuprofen ausgelöst wurde.

Erythema Exsudativum Multiforme Majus with Predominant Mucous Membrane Involvement (Fuchs' Syndrome) Triggered by Ibuprofen. Fuchs' syndrome is a subtype of erythema exsudativum multiforme majus (EEMM). We report on a 46-year old woman who developed this severe disease pattern following Ibuprofen medication.

\section{Einleitung}

Nach der heutigen Klassifikation werden schwere bullöse Hautreaktionen unterschieden in Erythema exsudativum multiforme majus (EEMM), Stevens-Johnson-Syndrom (SJS), Überlappungssyndrom (EEMM-SJS) sowie toxische epidermale Nekrolyse (TEN), wobei die Zuordnung im Einzelfall weiterhin Schwierigkeiten bereiten kann [1 -3]. Das EEMM ist durch gestörtes Allgemeinbefinden mit Hauterscheinungen, welche $\mathrm{zu}$ hämorrhagischer und bullöser Entwicklung neigen, charakterisiert. Schleimhautbeteiligung ist dabei obligat. Eine sehr seltene Sonderform des EEMM mit ganz überwiegendem Schleimhautbefall ist das Fuchs-Syndrom, das vom Wiener Augenarzt E. Fuchs 1876 erstmals beschrieben wurde.

Wir berichten über einen schweren Erkrankungsfall, bei dem das Syndrom nach Einnahme von Ibuprofen auftrat und recherchieren über die Wahrscheinlichkeit eines solchen Ereignisses.

\author{
P. Gruner ${ }^{1}$, A. Happ', N. Haas ${ }^{2}$ \\ ${ }^{1}$ Hautklinik des Humaine Klinikums Bad Saarow \\ (Leiter: Dr. A. Happ) \\ ${ }^{2}$ Klinik für Dermatologie, Venerologie und Allergologie mit \\ Asthmapoliklinik, Universitätsklinikum Charité der \\ Humboldt-Universität Berlin (Direktor: Prof. Dr. W. Sterry)
}

\section{Fallbericht}

\section{Anamnese}

Eine 46-jährige Frau, die seit 2 Monaten an Diarrhöe litt, bemerkte 4 Tage vor der stationären Aufnahme eine schmerzhafte Entzündung am äußeren Genitale. Tags darauf entwickelten sich eine Stomatitis und eine Konjunktivitis mit rascher Progredienz. Das Allgemeinbefinden verschlechterte sich zunehmend und die Patientin wurde unter dem Verdacht einer Gingivostomatitis herpetica in die Hautklinik eingewiesen. Sie berichtete, in unregelmäßigen Abständen wegen Rückenschmerzen Dolormin ${ }^{\circledR}$ Brausetabletten (Ibuprofen) eingenommen $\mathrm{zu}$ haben, letztmalig eine Woche vor der stationären Aufnahme. Ein Herpes simplex wurde verneint.

\section{Aufnahmebefund}

Es bestand eine hämorrhagische Konjunktivitis mit Erosionen der Cornea (Abb. 1). Die Mundschleimhaut zeigte flächige Erosionen und Ulzerationen, die mit fibrinösen Membranen bedeckt waren (Abb. 2). Die Lippen wiesen Rhagaden und Blutkrusten auf. An der Genitalschleimhaut fanden sich aphthöse Ulzera und schmerzhafte flächige Erosionen. Bis auf zwei unscheinbare, blasse, urtikarielle Herde mit kokardenartigem Aspekt am linken Handgelenk war das Integument frei. Das Allgemeinbefinden war deutlich reduziert, es bestand ein ausgeprägtes Krankheitsgefühl.

Untersuchungsbefunde: Leukozytose von 11,0 Gpt/l. Transaminasenanstieg auf ASAT 1,03 ukat/l und ALAT 1,04 ukat/l. Deutliche Erhöhung der renalen Parameter wie Kreatinin auf $186 \mathrm{mmol} / \mathrm{l}$ und Harnstoff auf $17,2 \mathrm{mmol} / \mathrm{l}$ bei gleichzeitiger Leukozyturie, Erythrozyturie und Proteinurie von $3665 \mathrm{mg} / \mathrm{l}$. Eine Stuhluntersuchung auf okkultes Blut war negativ. Der kulturelle Nachweis von Herpes simplex von der Mundschleimhaut war negativ. Ebenso die serologischen Untersuchungen auf HSV 1 und 2, Hepatitis B, Chlamydien und Yersinien. Die röntgenologische Untersuchung zeigte einen altersentsprechenden, regelrechten Thoraxorganbefund. 


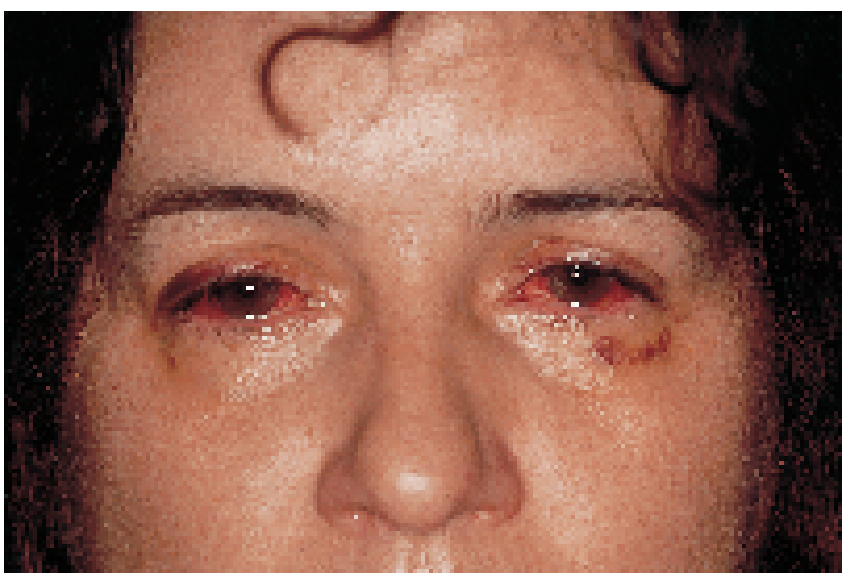

Abb.1 Hämorrhagische Konjunktivitis bei EEMM (Fuchs-Syndrom).

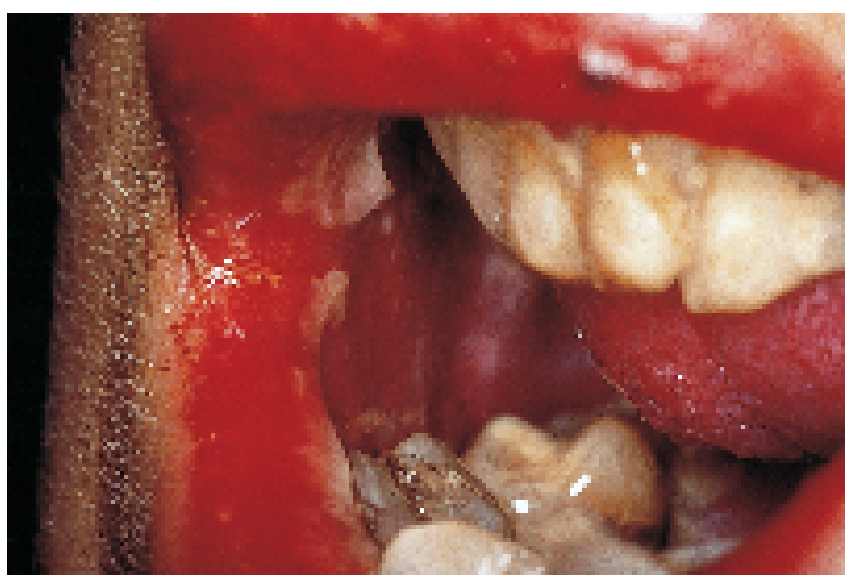

Abb. 2 Von fibrinösen Membranen bedeckte Ulzerationen der Mundschleimhaut und Lippen bei EEMM (Fuchs-Syndrom).

\section{Therapie und Verlauf}

Aufgrund des typischen Schleimhautbildes und der angedeuteten kokardenartigen Effloreszenzen am Handgelenk wurde die Diagnose des Fuchs-Syndroms gestellt. Die Patientin erhielt initial $250 \mathrm{mg}$ Prednisolon/d. Zusätzlich begannen wir eine Behandlung mit 3 × $250 \mathrm{mg}$ Aciclovir/d und Ciprofloxacin $2 \times 500 \mathrm{mg} / \mathrm{d}$. Lokal behandelten wir mit Povidon Jodlösung, Amphotericin Lutschtabletten, Lidocain Spray, 0,5\% Methylrosanilinumchlorid Lösung, Ol. Zinci, Dexamethason-, Aureomycin- und Panthenolaugensalbe.

Darunter kam es zu einer Progredienz der Schleimhautveränderungen. Das Allgemeinbefinden verschlechterte sich weiter, die Temperatur stieg bis $38,7^{\circ} \mathrm{C}$. Die eingeschränkte Nahrungs-und Flüssigkeitsaufnahme bei quälender Salivation erforderte eine parenterale Substitution. Wegen der sich verschlechternden Nierenparameter mit Makrohämaturie wurden die potenziell nephrotoxischen Medikamente Aciclovir und Ciprofloxacin abgesetzt. Ab dem 6. Tag stabilisierte sich der Allgemeinzustand, die Prednisolondosis konnte langsam reduziert werden, aber erst nach 5 Wochen kam es zu einer restitutio ad integrum.

\section{Diskussion}

Die Zuordnung der schweren bullösen Hautreaktionen zu den Krankheitsbildern EEMM, SJS und TEN wurde kürzlich durch eine Consensuskonferenz erleichtert [1]. Trotzdem ist es nicht immer einfach, einen Erkrankungsfall einzuordnen, denn fließende Übergänge kommen vor. Außerdem verbleiben historisch gut dokumentierte Sonderformen wie das FuchsSyndrom. Liegen die Hautveränderungen wie in unserem Fall im Hand- und Fußbereich, so ist es als eine Unterform des EEMM einzustufen [3].

Wurden früher vornehmlich Infekte als Auslöser angeschuldigt, so sieht man diese heute eher als Kofaktoren an, wobei weiterhin EEMM nach Infektionen ohne Medikamenteneinnahme beschrieben werden [2,4]. Infektionen bleiben aber differenzialdiagnostisch von Bedeutung, wie die Herpesstomatitis, die in unserem Fall vor schwierige therapeutische Entscheidungen stellte. Mittlerweile steht die Auslösung durch Arzneimittel ganz im Vordergrund der pathogenetischen Überlegungen. Man nimmt an, dass es durch Pharmaka zu Oberflächenveränderungen an den Keratinozyten mit anschließender Zerstörung kommt [5].

Die Reihenfolge der angeschuldigten Medikamente wird wie folgt angegeben: mit Abstand am häufigsten Antibiotika (Sulfonamide, Amoxicillin), dann Antiepileptika (Carbamacepin), erst dann folgen NSA und andere Stoffgruppen. Die Häufigkeit schwerster Reaktionen ist insgesamt selten: Sie wurde nach neuesten Erhebungen mit 2/Million Einwohner im Jahr angegeben [6].

Nach Untersuchungen in den USA, wo Ibuprofen frei verkäuflich ist, soll die Nebenwirkungsrate (an allen Organsystemen) nicht höher als bei Paracetamol liegen [7]. Von anderen Autoren wurde sie mit einer dokumentierten Nebenwirkung auf 5 25 Millionen verkaufte Tabletten angegeben [8]. Wir haben die Literatur daraufhin durchgesehen, ob ein EEMM nach Ibuprofen beschrieben wurde. In Zusammenstellungen über schwere Hautreaktionen aus Asien [9], den USA [4] und in der großen deutschen Studie [1], auch in augenärztlichen Berichten [10], fanden sich keine Berichte über Ibuprofen. In einer landesweiten italienischen Studie zu Hautreaktionen auf Analgetika/Antipyretika und NSA fanden sich unter 354 Fällen lediglich je ein Bericht über ein EEM und ein SJS nach Ibuprofen [11]. Eine zusammenfassende Dokumentation aus Frankreich, Deutschland, Italien und Portugal über 245 Fälle von TEN und SJS verzeichnete über die beiden aus Italien berichteten Fälle hinaus keine weiteren schweren Hautreaktionen nach Ibuprofen [12]. Ein Fuchs-Syndrom nach diesem Medikament dürfte demnach ein äußerst seltenes Ereignis sein. Aufgrund des schweren Verlaufes verzichteten wir auf eine Reexposition und bescheinigten eine Unverträglichkeit auf Arylpropionsäurederivate.

Wie in der Literatur beschrieben, war auch bei unserer Patientin trotz sofortiger, adäquater Therapie der klinische Verlauf nicht vorhersehbar [3]. Komplikationen wie tubuläre Nekrosen mit Nierenversagen, Pneumonien oder toxisches Kreislaufversagen traten glücklicherweise jedoch nicht auf. 


\section{Literatur}

${ }^{1}$ Mockenhaupt M, Schöpf E. Epidemiology of drug-induced severe skin reactions. Semin Cutan Med Surg 1996; 15: 236-243

2 Petter G, Haustein UF. Stevens-Johnson-Syndrom mit Übergang in eine toxisch epidermale Nekrolyse nach Carbamazepin-Einnahme, Heroin- und Alkoholabusus. Hautarzt 1999; 5: 884-888

${ }^{3}$ Rzany B, Mockenhaupt M, Baur S, Stocker U, Schöpf E. Schwere Hautreaktionen. Hautarzt 1993; 44: 549-555

${ }^{4}$ Chan HL, Stern RS, Arndt KA, Langlois J, Jick SS, Jick H, Walker AM. The incidence of erythema multiforme, Stevens-Johnson syndrome and toxic epidermal necrolysis. A population-based study with particular reference to reactions caused by drugs among outpatients. Arch Dermatol 1990; 126: 43-47

${ }^{5}$ Bruynzeel I, Van der Raaij EM, Boorsma DM, De Haan P, Willemze R. Increased adherence to keratinocytes of peripheral blood mononuclear leucocytes of a patient with drug-induced erythema multiforme. Br J Dermatol 1993; 129: 45-49

${ }^{6}$ Rzany B, Mockenhaupt M, Baur S, Schroder W, Stocker U, Mueller J, Hollander N, Bruppacher R, Schöpf E. Epidemiology of erythema exsudativum multiforme majus, Stevens-Johnson syndrome, and toxic epidermal necrolysis in Germany (1990-1992): structure and results of a population-based registry. J Clin Epidemiol 1996; 49: $769-773$

${ }^{7}$ Johnson JH, Jick H, Hunter JR, Dickson JF. A follow-up study of ibuprofen users. J Rheumatol 1985; 12: 549-552

${ }^{8}$ Moore N, Noblet C, Breemeersch C. Mise au point sur la securité de l'ibuprofene a dose antalgique-antipyretique. Therapie 1996; 51: $458-463$

${ }^{9}$ Chan HL. Observations on drug-induced toxic epidermal necrolysis in Singapore. J Am Acad Dermatol 1984; 1: 973 - 978

${ }^{10}$ Kuper K, Pleyer U, Zierhut M, Thiel HJ. Erythema exsudativum multiforme majus. Ophthalmologe 1995; 92: 823-888

${ }^{11}$ Gruppo Italiano Studi Epidemiologici in Dermatologia. Cutaneous reactions to analgesic-antipyretics and nonsteroidal antiinflammatory drugs. Dermatology 1993; 186: 164-169

${ }^{12}$ Roujeau JC, Kelly JP, Naldi L, Rzany B, Stern RS, Anderson T, Auquier A, Bastuji Garin S, Correia O, Locati F, et al. Medication use and the risk of Stevens-Johnson syndrome or toxic epidermal necrolysis. N Engl J Med 1995; 333: 1600-1607

\section{PD Dr. N. Haas}

Klinik für Dermatologie, Venerologie und Allergologie mit Asthmapoliklinik, Universitätsklinikum Charité der Humboldt-Universität Berlin

Schumannstraße 20/21

10117 Berlin

\section{MITTEILUNG}

\section{Berliner Stiftung für Dermatologie}

Die Berliner Stiftung für Dermatologie wurde vor wenigen Jahren gegründet, ausgehend von dem gemeinnützigen Verein 17. Dermatologischer Weltkongress e.V., der diesen Kongress 1987 in Berlin erfolgreich durchgeführt hat. Eine finanzielle Unterstützung hat dankbarerweise auch die Fa. Schering AG, Berlin, zur Verfügung gestellt. Die nach dem deutschen Gesetz anerkannte und rechtlich selbstständige Stiftung hat ihren Sitz zurzeit an der Klinik und Poliklinik für Dermatologie der Freien Universität Berlin und sich die Aufgabe gestellt, die Wissenschaft auf dem Gebiet der Dermatologie und ihrer Grenzgebiete zu fördern.

Aufgaben und finanzielle Mittel: Gemäß ihrer Satzung hat die Stiftung als besonderes Ziel, finanzielle Ressourcen zu bündeln und die dermatologische Forschung, insbesondere auf dem Gebiet der Molekularbiologie und der Dermatopharmakologie, voranzutreiben. Ebenso soll die Umsetzung von neuen Erkenntnissen zur Diagnose und Behandlung von Hautkrankheiten in der Klinik gefördert werden. Das Stiftungskapital beträgt derzeit ca. 1350000 DM; davon steht eine jährliche Förderungssumme von ca. 50000 DM für zeitlich befristete Forschungsvorhaben klinischer und experimenteller Art, die jeweils durch das Kuratorium geprüft werden, zur Verfügung. Ein weiteres Ziel der Stiftung ist es, den internationalen Austausch von Wissenschaftlern auf dem Gebiet der dermatologischen Forschung zu fördern; dazu werden beispielsweise Stipendien für eine Weiterbildung in Deutschland über eine befristete Zeit vergeben.

Eine wichtige Aufgabe der Berliner Stiftung ist die Vergabe eines Stiftungspreises - mindestens alle 2 Jahre - in Höhe von 25000 DM an jüngere Wissenschaftlerinnen und Wissenschaftler, die sich aufgrund persönlicher und wissenschaftlicher Leistungen in der Dermatologie hochrangig qualifiziert haben. Vorschläge für den Stiftungspreis und/oder für eine Unterstützung geeigneter wissenschaftlicher Projekte (Stipendien, Forschungsprogramme, Seminare) sind zu richten an das Kuratorium über seinen Geschäftsführer, Herrn Prof. Dr. Dr. C. C. Geilen oder seinen Stellvertreter, Herrn Prof. Dr. C. E. Orfanos, beide in Berlin. Formlose Bewerbungen sind möglich, doch Anträge und weitere Informationen sind im Sekretariat oder über die Homepage der Stiftung zu erhalten, um den Vorgang zu vereinfachen (Adressen siehe unten).

Nominierungsvorschlägen für den jährlichen Stiftungspreis sollte möglichst das Kurrikulum und die neueste Publikationsliste des Kandidaten beigefügt werden. Anträge werden jährlich vorzugsweise in den Monaten Januar bis August angenommen und für das laufende Jahr ausgewertet, später eingegangene Anträge werden für die Mittelvergabe im folgenden Jahr berücksichtigt. Die Bekanntgabe der Preisträger bzw. der Stipendiaten erfolgt in der Regel vor dem 15. November.

Kuratorium: Priv.-Doz. Dr. Dr. Christoph C. Geilen (Geschäftsführer), Prof. Dr. Harald P. M. Gollnick, Klaus von der Heyde, Prof. Dr. Constantin E. Orfanos, Prof. Dr. Günter Stüttgen, Prof. Dr. Thomas Trautner; Stiftungssekretariat c/o Klinik und Poliklinik für Dermatologie, Fabeckstr. 60 -62, 14195 Berlin, Tel. 030/8445-6907, Fax 030/8445-6907, www.stiftung-dermatologie.de 